\title{
P052: Molecular epidemiology of methicillin-resistant Staphylococcus aureus (MRSA) strains at Geneva University Hospitals (HUG) over a 9 year period
}

\author{
C Fankhauser $^{1 *}$, J Schrenzel ${ }^{2}$, P François ${ }^{3}$, G Renzi ${ }^{2}$, D Pittet ${ }^{1}$, S Harbarth ${ }^{1}$ \\ From 2nd International Conference on Prevention and Infection Control (ICPIC 2013) \\ Geneva, Switzerland. 25-28 June 2013
}

\begin{abstract}
Introduction
Changes within the MRSA population of single hospitals have been observed with certain clones replacing others. Surveillance of the genetic diversity within a hospital provides useful epidemiological data. Staphylococcal chromosomic cassettes (SCCmec) of MRSA isolates are routinely determined at HUG.
\end{abstract}

\section{Objectives}

To describe secular trends of the predominant MRSA clones retrieved at HUG from 2003-12 using SCCmec genotyping.

\section{Methods}

Since 2005, the SCCmec in MRSA isolates (screening swabs or clinical samples) were routinely assessed by multiplex PCR assay. MLVA was used to evaluate genomic diversity; representative isolates were grouped in MLVA clusters and subjected to MLST. All patients' $1^{\text {st }}$ positive MRSA isolate/year was analysed. Frequency distributions and hospital-acquired (HA)-MRSA rates were determined.

\section{Results}

HA-MRSA cases/100 admissions increased from 1.36 (2000) to 2.00 (2006) and declined to 0.79 (2012). Overall, 9717 MRSA isolates underwent SCCmec genotyping. SCCmecI, the predominant cassette during the study period, peaked at $88 \%$ in 2003 and declined to $64 \%$ in 2012 . SCCmecIV increased modestly from $10 \%$ to $16 \%$, followed by SCCmecII with a strong increasing trend from $2 \%$ to $14 \%$. Other types were minor contributors (SCCmecV, $3.4 \%$; others $<2 \%)$. Patients harboring SCCmecI and

$\overline{1}$ Infection Control Program, Geneva University Hospitals, Geneva, Switzerland Full list of author information is available at the end of the article
SCCmecII were older (median age, 76 and 82 y) compared to those with SCCmecIV (60 y). Strain distribution differed by hospital sectors. While SCCmecI remained the prevalent subtype in acute care $(\mathrm{AC})$ and non-AC settings, the proportion of MRSA containing SCCmecII and SCCmecIV was higher in non-AC settings. SCCmecII increased from $6 \%$ to $32 \%$ in geriatrics. Genotyping confirmed ST228 South German-SCCmecI as the predominant clone. ST105 (CC5) appears to be the predominant clone of SCCmecII. Individual SCCmec replacement was observed in 123 patients (incl. 37 within SCCmecISCCmecII and 69, SCCmecI-SCCmecIV).

\section{Conclusion}

ST 228 SCCmecI is still the predominant clone in all settings, but decaying. The prevalence of SCCmecII is higher in geriatrics than in AC settings. The emerging SCCmecII predominant clone is ST105(CC5).

\section{Disclosure of interest}

None declared.

\section{Author details}

${ }^{1}$ Infection Control Program, Geneva University Hospitals, Geneva, Switzerland. ${ }^{2}$ Bacteriology Laboratory, Geneva University Hospitals, Geneva, Switzerland. ${ }^{3}$ Genomic Research Laboratory, Geneva University Hospitals, Geneva, Switzerland.

Published: 20 June 2013

doi:10.1186/2047-2994-2-S1-P52

Cite this article as: Fankhauser et al.: P052: Molecular epidemiology of methicillin-resistant Staphylococcus aureus (MRSA) strains at Geneva

University Hospitals (HUG) over a 9 year period. Antimicrobial Resistance and Infection Control 2013 2(Suppl 1):P52.

\section{C) Biomed Central}

(c) 2013 Fankhauser et al; licensee BioMed Central Ltd. This is an Open Access article distributed under the terms of the Creative Commons Attribution License (http://creativecommons.org/licenses/by/2.0), which permits unrestricted use, distribution, and reproduction in any medium, provided the original work is properly cited. 